ISSN 1855-3966 (printed edn.), ISSN 1855-3974 (electronic edn.)

ARS MATHEMATICA CONTEMPORANEA 17 (2019) 103-114

https://doi.org/10.26493/1855-3974.1740.803

(Also available at http://amc-journal.eu)

\title{
Integral regular net-balanced signed graphs with vertex degree at most four
}

\author{
Zoran Stanić * \\ Faculty of Mathematics, University of Belgrade, \\ Studentski trg 16, 11000 Belgrade, Serbia
}

Received 29 June 2018, accepted 14 January 2019, published online 22 August 2019

\begin{abstract}
A signed graph is called integral if its spectrum consists entirely of integers, it is $r$-regular if its underlying graph is regular of degree $r$, and it is net-balanced if the difference between positive and negative vertex degree is a constant on the vertex set (this constant is called the net-balance and denoted $\varrho$ ). We determine all the connected integral 3 -regular net-balanced signed graphs. In the next natural step, for $r=4$, we consider only those whose net-balance is a simple eigenvalue. There, we complete the list of feasible spectra in bipartite case for $\varrho \neq 0$ and prove the non-existence for $\varrho=0$. Certain existence conditions are established and the existence of some 4-regular (simple) graphs is confirmed. In this study we transferred some results from the theory of graph spectra; in particular, we give a counterpart to the Hoffman polynomial.
\end{abstract}

Keywords: Signed graph, switching equivalent signed graphs, adjacency matrix, net-balanced signed graph.

Math. Subj. Class.: 05C50, 05C22

\section{Introduction}

A signed graph $\dot{G}$ is obtained from a (simple) graph $G$ by accompanying each edge $e$ by the sign $\sigma(e) \in\{1,-1\}$ (chosen in any way for any edge). The (multiplicative) sign group $\{1,-1\}$ can also be written $\{+,-\}$. We say that $G$ is the underlying graph of $\dot{G}$. The set of vertices of $\dot{G}$ is denoted $V(\dot{G})$. The number of vertices and the number of edges of $\dot{G}$ are denoted $n$ and $m$, respectively. Clearly, every graph can be interpreted as a signed graph.

*This research is partially supported by the Serbian Ministry of Education, Science and Technological Development, Projects 174033 and 174012. The author is grateful to the anonymous referee for valuable suggestions.

E-mail address: zstanic@math.rs (Zoran Stanić) 
The $n \times n$ adjacency matrix $A_{\dot{G}}$ of $\dot{G}$ is obtained from the standard (0,1)-adjacency matrix of $G$ by reversing the sign of all 1's which correspond to negative edges. The eigenvalues of $A_{\dot{G}}$ are real and form the spectrum of $\dot{G}$. A detailed introduction to spectra of signed graphs can be found in [7, 10].

A graph is integral if its spectrum consists entirely of integers. The problem of identifying such graphs was posed by Harary and Schwenk [3] in 1974. Since then, a number of results concerning integral graphs have appeared in various references, including research articles, thesis and book chapters (not listed here).

Integral signed graphs are defined in the same way. Transferring the problem to the domain of signed graphs - to the author's knowledge, no one has considered this problem for signed graphs - results in various solutions, some of them having interesting and, at first glance, interesting properties. For example, the signed graph illustrated in Figure 1 is integral and has only two eigenvalues: 2 and -2 (both with multiplicity 3 ). Moreover, every switching equivalent signed graph is also integral (since it has the same spectrum; see the next section for the details).

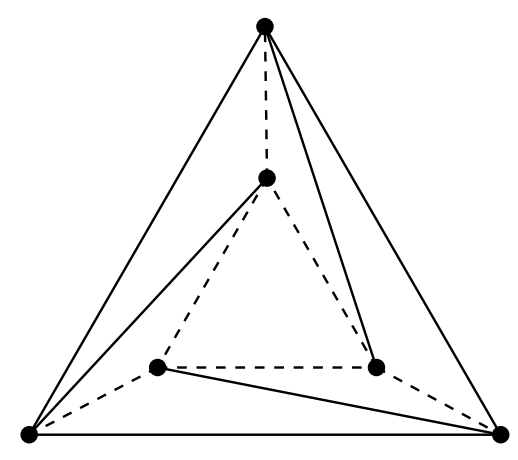

Figure 1: An integral signed graph. (Here and following, negative edges are dashed.)

Our results are announced in the abstract. In Section 2 we introduce the terminology and notation, and give some preliminary results. Sections 3 and 4 are devoted to integral signed graphs which are 3-regular and 4-regular, respectively. An existence condition and certain integral 4-regular (simple) graphs are established in Section 5.

\section{Preliminaries}

We write $d_{i}^{+}$and $d_{i}^{-}$for the positive and negative vertex degree (i.e., the number of positive and negative edges incident with $i$ ). The existence of a positive (resp. negative) edge

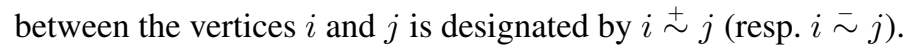

A walk in a signed graph is a sequence of alternate vertices and edges such that the consecutive vertices are endpoints of the corresponding edge. A walk is positive if the number of its negative edges (with possible repetitions) is not odd. Otherwise, it is negative. Since every cycle in a signed graph is a walk, we may talk about positive or negative cycles, as well.

We say that a signed graph is bipartite or regular (of degree $r$ ) if the same holds for its underlying graph. (There is a different approach for regularity in [10].) The spectrum of a bipartite signed graph is symmetric with respect to the origin. The net-balance of a vertex 
$i$ is defined by $d_{i}^{+}-d_{i}^{-}$. We also say that a signed graph is net-balanced if the net-balance is a constant on the vertex set; in that case the net-balance is denoted $\varrho$.

For $U \subset V(\dot{G})$, let $\dot{G}^{U}$ be the signed graph obtained from $\dot{G}$ by reversing the sign of each edge between a vertex in $U$ and a vertex in $V(\dot{G}) \backslash U$. The signed graph $\dot{G}^{U}$ is said to be switching equivalent to $\dot{G}$. Switching equivalent signed graphs share the same spectrum.

The reverse $\operatorname{rev}(\dot{G})$ of $\dot{G}$ is obtained by reversing the sign of all edges of $\dot{G}$.

We use the following facts without proofs (for details, see [8, 10]):

- The eigenvalues of $\operatorname{rev}(\dot{G})$ (with repetitions) are obtained by reversing the sign of the eigenvalues of $\dot{G}$.

- Signed graphs $\dot{G}$ and $\operatorname{rev}(\dot{G})$ are switching equivalent if and only if $\dot{G}$ is bipartite.

- A signed graph $\dot{G}$ is switching equivalent to $G$ (the underlying graph) if and only if the vertices of $\dot{G}$ can be divided into two sets (one of them possibly empty) in such a way that an edge is negative if and only if it joins vertices from different sets.

- The spectrum of every net-balanced signed graph contains its net-balance.

It is known that the largest eigenvalue $\rho$ of a signed graph does not exceed the largest eigenvalue of its underlying graph. The proof follows by the next chain of (in)equalities derived on the basis of the Rayleigh principle:

$$
\rho(\dot{G})=2\left(\sum_{\substack{\sim \\ i \sim j}} x_{i} x_{j}-\sum_{i \bar{\sim} j} x_{i} x_{j}\right) \leq 2\left(\sum_{\substack{i \sim j \\ \sim}}\left|x_{i} x_{j}\right|+\sum_{i \bar{\sim}_{j}}\left|x_{i} x_{j}\right|\right) \leq \rho(G),
$$

where $\mathbf{x}=\left(x_{1}, x_{2}, \ldots, x_{n}\right)^{T}$ is a unit eigenvector associated with $\rho(\dot{G})$. What we need here is the opposite implication.

Lemma 2.1. For a connected signed graph $\dot{G}$, if $\rho(\dot{G})=\rho(G)$ then $\dot{G}$ and $G$ are switching equivalent.

Proof. Since $\rho(\dot{G})=\rho(G)$, all the inequalities in (2.1) reduce to equalities. This, in particular, means that $|\mathbf{x}|$ is an eigenvector associated with $\rho(G)$, and so it holds $x_{i} \neq 0$, for all $i$. Applying switching with respect to the set of vertices that correspond to negative coordinates of $\mathbf{x}$, we arrive at the signed graph, say $\dot{H}$, such that $|\mathbf{x}|$ is associated with $\rho(\dot{H})$, as well. (The matrix transformation is realized by $A_{\dot{H}}=D^{-1} A_{\dot{G}} D, D$ being the diagonal matrix of \pm 1 where the sign of a diagonal entry is determined by the sign of the corresponding coordinate of $\mathbf{x}$. Then, $|\mathbf{x}|=D \mathbf{x}$.)

Since $\rho(\dot{H})=\rho(G)$, it follows (by (2.1), with $\dot{H}$ and $|\mathbf{x}|$ in the roles of $\dot{G}$ and $\mathbf{x}$ ) that $\dot{H}$ does not contain negative edges, i.e., $\dot{H}$ is a graph isomorphic to $G$, and we are done.

Connected integral regular net-balanced signed graphs of vertex degree 0,1 or 2 are easily determined. In what follows, we move up to $r=3$ and $r=4$.

Obviously, if $\dot{G}$ is an integral net-balanced signed graph with $\varrho \geq 0$, then the reverse $\operatorname{rev}(\dot{G})$ is also integral net-balanced with $\varrho \leq 0$, and vice versa. Thus, it is sufficient to consider only those with $\varrho \geq 0$. 


\section{Case $r=3$}

Recall that connected 3-regular graphs are determined in [2, 4]; there are 13 such graphs. In what follows, we refer to the notation of the latter reference.

By Lemma 2.1, if an $r$-regular signed graph $\dot{G}$ has $r$ as an eigenvalue, then $\dot{G}$ is switching equivalent to its underlying graph. If $r$ does not belong to the spectrum of $\dot{G}$, but $-r$ does, then $\operatorname{rev}(\dot{G})$ is switching equivalent to its underlying graph. This observation leads to the following result.

Theorem 3.1. If a connected 3-regular net-balanced signed graph $\dot{G}$ of non-negative net-balance $\varrho$ is integral and at least one of the numbers 3 or -3 is its eigenvalue, then $\dot{G}$ is determined in the following way.

(a) If 3 is an eigenvalue of $\dot{G}$, then

(i) for $\varrho=3, \dot{G}$ is one of the 13 connected 3 -regular graphs $G_{1}, \ldots, G_{13}$ obtained in $[4]$;

(ii) for $\varrho=1, \dot{G}$ is switching equivalent to one of the graphs $G_{2}, G_{4}, G_{5}, G_{7}, G_{10}$ or $G_{12}$ of [4].

(b) If 3 is not an eigenvalue of $\dot{G}$, then

(i) case $\varrho=3$ cannot occur;

(ii) for $\varrho=1, \dot{G}$ is switching equivalent to $G_{9}$ or $G_{13}$ of [4].

Proof. (a): By Lemma 2.1, $\dot{G}$ is switching equivalent to its underlying graph, and then (a.i) follows directly by the result of the corresponding reference.

(a.ii): Here, $\dot{G}$ is obtained by identifying a perfect matching in one of $G_{1}, \ldots, G_{13}$ and reversing the sign of all edges in the matching. In addition, this reversing must produce a switching equivalent graph. In particular, this means that the vertex set can be partitioned into two sets such that an edge is negative if and only if it joins vertices from different sets. Inspecting all 13 graphs, we conclude that such an action can be performed for the 6 graphs listed. (The procedure is simplified by excluding the signed graphs which do not have the net-balance as an eigenvalue.)

(b): Case (b.i) follows directly.

(b.ii): Here, $\dot{G}$ is non-bipartite and 3 is an eigenvalue of $\operatorname{rev}(\dot{G})$. Considering $G_{9}, \ldots, G_{13}$ as candidates for a graph which is switching equivalent to $\operatorname{rev}(\dot{G})$, we arrive at the 2 solutions: $G_{9}$ and $G_{13}$.

A transfer of a result from the domain of simple graphs is needed. For signed graphs $\dot{G}_{1}$ and $\dot{G}_{2}$, the (tensor) product $\dot{G}_{1} \times \dot{G}_{2}$ is the signed graph with the vertex set $V\left(\dot{G}_{1}\right) \times V\left(\dot{G}_{2}\right)$ in which two vertices $\left(u_{1}, u_{2}\right)$ and $\left(v_{1}, v_{2}\right)$ are adjacent if and only if $u_{i}$ and $v_{i}$ are adjacent in $\dot{G}_{i}$, for $1 \leq i \leq 2$. The sign of an edge of $\dot{G}_{1} \times \dot{G}_{2}$ is equal to the product of signs of the corresponding edges of $\dot{G}_{1}$ and $\dot{G}_{2}$. The adjacency matrix of $\dot{G}_{1} \times \dot{G}_{2}$ is then identified with the Kronecker product $A_{\dot{G}_{1}} \otimes A_{\dot{G}_{2}}$. Accordingly, if $\lambda_{1}, \lambda_{2}, \ldots, \lambda_{n}$ are the eigenvalues of $\dot{G}_{1}$ and $\mu_{1}, \mu_{2}, \ldots, \mu_{m}$ are the eigenvalues of $\dot{G}_{2}$, then the eigenvalues of their product are $\lambda_{i} \mu_{j}(1 \leq i \leq n, 1 \leq j \leq m)$. In particular, if $\dot{G}$ is a connected integral non-bipartite signed graph, then $\dot{G} \times K_{2}$ is a connected integral bipartite signed graph, since the eigenvalues of $K_{2}$ are 1 and -1 . The signed graph $\dot{G} \times K_{2}$ is called a bipartite double (of $\dot{G}$ ). 


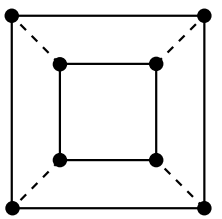

$\dot{G}_{2}$

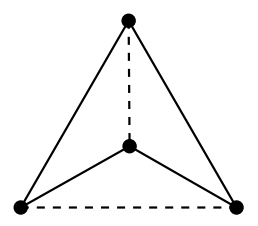

$\dot{G}_{9}$

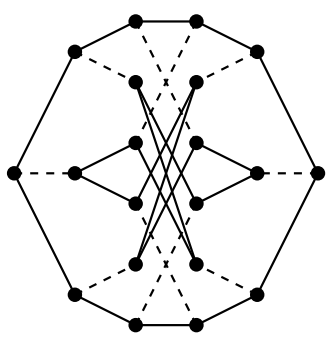

$\dot{G}_{4}$

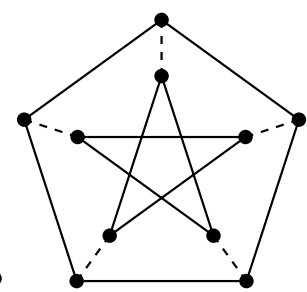

$\dot{G}_{10}$
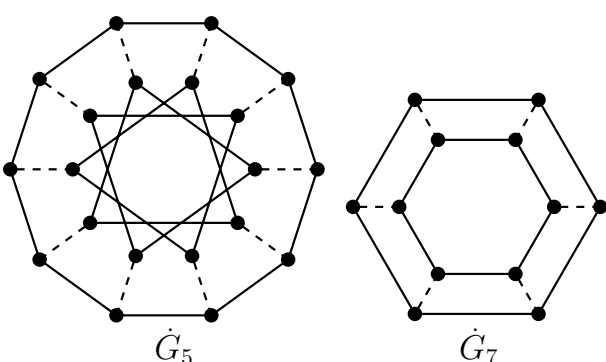

$\dot{G}_{7}$

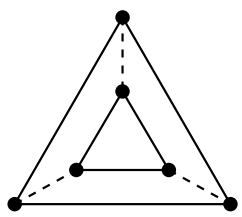

$\dot{G}_{12}$

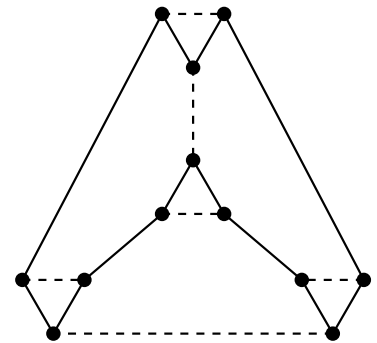

$\dot{G}_{13}$

Figure 2: Representatives of signed graphs of Theorem 3.1(a.ii)\&(b.ii).

Therefore, any non-bipartite signed graph $\dot{G}$ can be extracted from the decompositions of bipartite ones having the form $\dot{G} \times K_{2}$, and so the essential part in determining connected integral signed graphs consists of searching for those that are bipartite. If, in addition, a signed graph is regular then it has the same number of vertices in each colour class, and we may assume that the number of its vertices is $2 n$.

Returning to connected integral 3-regular net-balanced signed graphs, it remains to determine those that avoid \pm 3 in the spectrum. According to the previous observation, we may consider the bipartite ones, so those with $2 n$ vertices and the spectrum

$$
\left[2^{m_{2}}, 1^{m 1}, 0^{2 m_{0}},(-1)^{m_{1}},(-2)^{m_{2}}\right],
$$

where the exponents stand for the multiplicities.

Counting the difference between the numbers of positive and negative closed walks and considering the spectral moments, we get $m_{2}+m_{1}+m_{0}=n, 4 m_{2}+m_{1}=3 n$ and $16 m_{2}+m_{1}=15 n+4 q$, where $q$ is the difference between the numbers of positive and negative quadrangles contained.

At this point, one could continue by the spectral moments of higher order to obtain the feasible spectra, but this situation can easily be resolved in a different way. Solving the previous system, we get $m_{1}=-\left(n+\frac{4}{3} q\right)$ (and certain parametrizations of other multiplicities which are not important). The last equality implies that $q$ must be negative, that is our signed graph, say $\dot{G}$, must contain a negative quadrangle. If every vertex is at distance at most 1 from such a quadrangle, then $\dot{G}$ has at most 8 vertices. Otherwise, if there exists a vertex at distance 2 from a fixed negative quadrangle, then the vertex between them is adjacent to only one vertex of the quadrangle (otherwise, the largest eigenvalue of that signed subgraph would be greater than 2 , and then the same would hold for $\dot{G}$ as follows by the 
interlacing argument). So, $\dot{G}$ contains a negative quadrangle with a pendant path of length 2. Considering the possible neighbourhoods of the vertices of such a subgraph and bearing in mind the other conditions (3-regularity and net-balancedness), we conclude by hand that the largest eigenvalue of $\dot{G}$ must be greater than 2 .

Therefore, it remains to consider connected signed graphs with at most 8 vertices. This is easily performed by reversing the signs of edges of connected bipartite 3-regular graphs with 4, 6 and 8 vertices (in each case, there is only one such graph). Obviously, the net-balance cannot be equal to 3 , and since $\dot{G}$ is bipartite, it cannot be equal to -3 , either. The result is summarized in the next theorem.

Theorem 3.2. If $\dot{G}$ is a connected integral bipartite 3-regular net-balanced signed graph avoiding \pm 3 in the spectrum, then $\dot{G}$ is the signed graph illustrated in Figure 3 or its reverse.

Clearly, signed graphs obtained in the previous theorem are switching equivalent and none of them is a bipartite double.

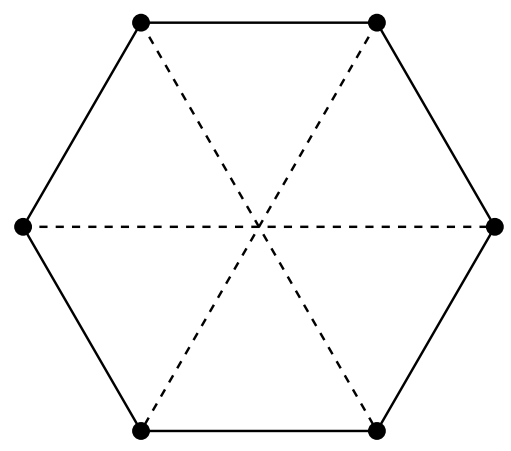

Figure 3: Integral signed graph from Theorem 3.2.

\section{Case $r=4$}

The next natural case is made up of connected integral 4-regular net-balanced signed graphs. We first recall that connected integral 4-regular graphs (so, signed graphs with net-balance equal to 4 ) are not fully determined. What we do know are the feasible spectra of such bipartite graphs, and [9] lists 828 such spectra (in the future this number could be decreased). The existence of the corresponding graphs is confirmed for a small number of those spectra - by the same reference, in 19 cases; in the next section we confirm 2 more.

Accordingly, it would be illusory to expect all the integral bipartite 4-regular signed graphs to be identified, even if we impose that they are net-balanced. Considering the feasible spectra, if \pm 4 is an eigenvalue, then they are the same as those listed in [9]. For an integral 4-regular graph, the corresponding signed graphs can be obtained as in Theorem 3.1. This will not be performed here; instead, we determine the feasible spectra of our signed graphs that avoid \pm 4 in the spectrum, but with the additional condition that they contain the net-balance as a simple eigenvalue. It occurs that there is a comparatively small number of such spectra. Before that, we prove a 'signed' variant of the result concerning the Hoffman polynomial of a graph [6, Theorem 2.1.6]. 
Theorem 4.1. For a signed graph $\dot{G}$, if there exists a polynomial $P$ such that $P\left(A_{\dot{G}}\right)=J$ ( $J$ being an all-1 matrix), then $\dot{G}$ is a connected net-balanced signed graph.

Conversely, if in addition the net-balance $\varrho$ is a simple eigenvalue of $\dot{G}$, then the polynomial exists and has the form

$$
P(x)=n \prod \frac{x-\lambda}{\varrho-\lambda},
$$

where $n$ is the number of vertices and the product goes over all distinct eigenvalues, excluding $\varrho$.

Proof. The proof is similar to that of the original result, along with certain adaptations tailored for signed graphs. For the sake of completeness, we give all the details. Denote $A=A_{\dot{G}}$. The existence of $P$ implies the identity $A J=J A$. Since the $(i, j)$-entry of $A J$ is $d_{i}^{+}-d_{i}^{-}$and the same entry of $J A$ is $d_{j}^{+}-d_{j}^{-}$, we have that $d_{i}^{+}-d_{i}^{-}$is a constant on the vertex set.

Clearly, $\dot{G}$ cannot be disconnected, since for $i$ and $j$ belonging to different components the $(i, j)$-entry of any power of $A$ would be zero, giving $P(A) \neq J$.

Denote $W(x)=\frac{\mu(x)}{x-\varrho}$, where $\mu$ stands for the minimal polynomial of $A$. As $\mu(A)=O$, it follows $(A-\varrho I) W(A)=O$, giving $A W(A)=\varrho W(A)$. Now, the unit vector $\mathbf{j}$ is an eigenvector associated with $\varrho$, but since this is the simple eigenvalue, the dimension of its eigenspace is 1 , and by the last identity, every column of $W(A)$ is a multiple of $\mathbf{j}$. Moreover, the symmetry of $W(A)$ implies

$$
W(A)=c J,
$$

for some $c \neq 0$. Thus, the polynomial exists and, so far, has the form $P(x)=\frac{1}{c} W(x)$.

Further, the identity $a_{k} A^{k} \mathbf{j}=a_{k} \varrho^{k} \mathbf{j}$ (for a real $a_{k}$ ) yields $W(A) \mathbf{j}=W(\varrho) \mathbf{j}$, which together with (4.2) gives $c J \mathbf{j}=W(\varrho) \mathbf{j}$, i.e., $n c=W(\varrho)$, which finally gives (4.1).

This result covers net-balanced signed graphs which need not be regular. The converse statement requires the multiplicity of $\varrho$ to be 1 , which will be an essential condition in our further considerations. Another consequence of (4.1), when $\dot{G}$ is additionally integral, is that $n$ divides the product $\prod(\varrho-\lambda)$; this condition was exploited in many searches for integral regular graphs.

Let

$$
\left[3^{m_{3}}, 2^{m_{2}}, 1^{m_{1}}, 0^{2 m_{0}},(-1)^{m_{1}},(-2)^{m_{2}},(-3)^{m_{3}}\right]
$$

denote the spectrum of our connected bipartite signed graph that avoids the eigenvalues 4 and -4 .

Theorem 4.2. If $\dot{G}$ is a connected integral bipartite 4-regular signed graph with $2 n$ vertices, then the multiplicities of its eigenvalues are parametrized by

$$
\begin{aligned}
m_{3} & =\frac{1}{180}(54 n+26 q+3 h), & m_{2} & =\frac{1}{30}(6 n-16 q-3 h), \\
m_{1} & =\frac{1}{4}(2 n+h)+\frac{5}{6} q, & m_{0} & =-\frac{1}{18}(8 q+3 h),
\end{aligned}
$$

where $q$ and $h$ respectively denote the difference between the numbers of positive and negative quadrangles and hexagons contained. 
Proof. Considering the spectral moments up to the 6 th order and counting the differences between the numbers of positive and negative closed walks, we arrive at the following Diophantine system:

$$
\begin{aligned}
& \sum_{i=-3}^{3} m_{|i|} i^{0}=2 n, \\
& \sum_{i=-3}^{3} m_{|i|} i^{2}=8 n, \\
& \sum_{i=-3}^{3} m_{|i|} i^{4}=56 n+8 q, \\
& \sum_{i=-3}^{3} m_{|i|} i^{6}=464 n+144 q+12 h .
\end{aligned}
$$

where, say for the 3rd equation, the first term on the right-hand side counts closed walks of length 4 traversing along at most 2 distinct edges. Observe that such walks are positive independently of the edge signature. Similarly, the second term counts the same walks traversing along quadrangles.

Solving this system for $m_{3}, \ldots, m_{0}$, we get the result.

Table 1: Feasible parameters of signed graphs described in Theorem 4.3.

\begin{tabular}{rrrrrrrr}
$n$ & $m$ & $m_{3}$ & $m_{2}$ & $m_{1}$ & $m_{0}$ & $q$ & $h$ \\
\hline \hline 6 & 24 & 2 & 1 & 2 & 1 & 3 & -14 \\
10 & 40 & 4 & 1 & 0 & 5 & 15 & -70 \\
15 & 60 & 6 & 1 & 2 & 6 & 21 & -92 \\
20 & 80 & 8 & 1 & 4 & 7 & 27 & -114 \\
30 & 120 & 11 & 1 & 17 & 1 & 21 & -62 \\
30 & 120 & 12 & 1 & 8 & 9 & 39 & -158 \\
60 & 240 & 23 & 1 & 29 & 7 & 57 & -194 \\
60 & 240 & 24 & 1 & 20 & 15 & 75 & -290 \\
60 & 240 & 25 & 1 & 11 & 23 & 93 & -386 \\
60 & 240 & 26 & 1 & 2 & 31 & 111 & -482
\end{tabular}

Include now the announced condition.

Theorem 4.3. A connected integral bipartite 4-regular net-balanced signed graph whose net-balance is 2 and appears as a simple eigenvalue has one of the spectra shown in Table 1. Each row contains one half of the number of vertices, the number of edges, the multiplicities of positive eigenvalues, one half of the multiplicity of 0 and previously defined parameters $q$ and $h$.

Proof. Solving the system (4.3) for $m_{2}=1$, we get

$$
\begin{aligned}
& m_{3}=\frac{1}{18}(6 n+q-3), \\
& m_{1}=\frac{1}{2}(2 n-q-5), \\
& m_{0}=\frac{1}{9}(-3 n+4 q+15) \quad \text { and } \\
& h=2 n-\frac{16}{3} q-10 \text {. }
\end{aligned}
$$


Now, since the net-balance is a simple eigenvalue, it follows that $2 n$ divides

$$
\prod_{i \neq 2, i=-3}^{3}(2-i)=120
$$

giving a finite number of possibilities for $n$. Observing the expressions of $m_{1}$ and $m_{0}$, we conclude that $q$ satisfies

$$
\frac{3}{4}(n-5) \leq q \leq 2 n-5,
$$

giving a finite range for this parameter. Concerning the possible values for $n$ and using the previous inequalities, we arrive at solutions listed in the table.

The existence of the signed graph with data as in the first row is confirmed by hand. Namely, we have considered connected bipartite 4-regular graphs with 12 vertices (there are 4) and arguing as in the proof of Theorem 3.1(a.ii). The resulting solution is illustrated in Figure 4.

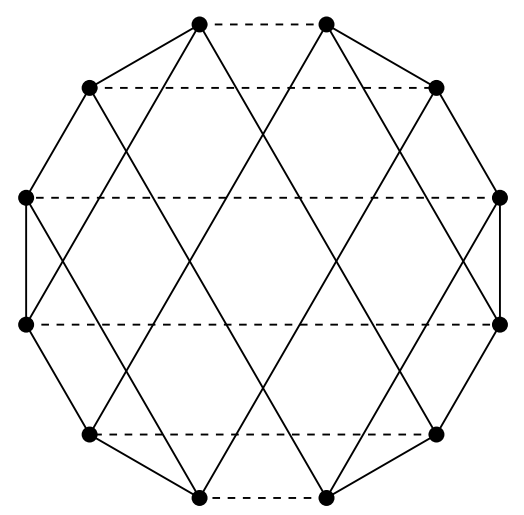

Figure 4: Integral signed graph with spectrum $\left[3^{2}, 2,1^{2}, 0^{2},(-1)^{2},-2,(-3)^{2}\right]$.

Remark 4.4. What about non-bipartite signed graphs? Let $\dot{G}$ be such a signed graph (with all the remaining conditions as in Theorem 4.3). Although the multiplicity of 2 in the spectrum of $\dot{G}$ is 1 , the same eigenvalue does not need to be simple in $\dot{G} \times K_{2}$. Therefore, the feasible parameters of the bipartite double are given by Theorem 4.2. The number of vertices (of $\dot{G} \times K_{2}$ ) now divides 240 , and the numbers of quadrangles and hexagons are bounded in terms of $n$ (see [1]) giving the magnitudes for our parameters $q$ and $h$. Therefore, the sets of feasible spectra can be obtained, but there are many, and we skip their presentation. Alternatively, one may consider the spectra of $\dot{G}$ directly, and include the odd spectral moments, but again, there are many.

Set now $\varrho=0$.

Theorem 4.5. There is no connected integral 4-regular net-balanced signed graph avoiding \pm 4 in the spectrum whose net-balance is 0 and appears as a simple eigenvalue. 
Proof. First, such a signed graph cannot be bipartite (see the multiplicity of 0). Let $\dot{G}$ be a non-bipartite signed graph described in this theorem (if any). Then the number of its vertices (denoted $n$ ) divides

$$
\prod_{i \neq 0, i=-3}^{3}(0-i)=36,
$$

i.e., the number of vertices of its bipartite double divides 72 . In addition, 0 is an eigenvalue of $\dot{G} \times K_{2}$ of multiplicity 2. Solving the system (4.3) for $m_{0}=2$ and every possible $n$, we arrive at exactly 9 solutions: one for $n=6$, one for $n=9$, one for $n=12$, 2 for $n=18$ and 4 for $n=36$.

There is another condition to be included: the parameter $q$ of $\dot{G} \times K_{2}$ cannot be odd. Indeed, by definition of the product, every quadrangle of $\dot{G}$ produces its two copies in $\dot{G} \times K_{2}$, and vice versa, every quadrangle of $\dot{G} \times K_{2}$ has a copy and both of them arise from the isomorphic quadrangle of $\dot{G}$. Therefore, the parameter $q$ of $\dot{G} \times K_{2}$ is twice the same parameter of $\dot{G}$, i.e., it cannot be odd.

Now, only the solution for $n=9$ passes this test for $q$ (with $q=-6$ ), which means that $\dot{G}$ could only have 9 vertices. Since for $\dot{G}, q=-3$, the number of quadrangles in the underlying graph $G$ must be odd, and this is not satisfied in the case of 6 (out of 16) connected 4-regular graphs with 9 vertices. For the remaining 10, one may choose between a computation by hand (which reduces to searching for cyclic decompositions and checking the spectra) or brute force performed by computer. In any case, there are no solutions (note that some of those underlying graphs produce signed graphs satisfying all but the last condition of the theorem - regarding the simplicity of 0 ).

Remark 4.6. In this section we restricted ourselves to net-balanced signed graphs whose net-balance appears as a simple eigenvalue. Of course, there are examples of those that are connected integral 4-regular and net-balanced, yet the net-balance is not a simple eigenvalue; at the end of the proof of Theorem 4.5, we mentioned that we met some of them. Another example is a net-balanced 4-dimensional cube with negative quadrangles. Indeed, its adjacency matrix can be written as

$$
\left(\begin{array}{cc}
A & A^{*} \\
A^{*} & A
\end{array}\right)
$$

where $A$ is the adjacency matrix of the cycle $C_{8}$ and $A^{*}$ is obtained from $A$ by reversing the sign of all the entries corresponding to 4 non-adjacent edges. We have $\varrho=2$, and the spectrum is given by $\left[2^{8},(-2)^{8}\right]$.

\section{An existence condition for bipartite regular graphs}

Following our idea of [5], we establish an existence condition for bipartite $r$-regular graphs with $q=0$. Namely, the adjacency matrix of such a graph can be written in the form

$$
A_{G}=\left(\begin{array}{cc}
O & B^{T} \\
B & O
\end{array}\right)
$$

and then the top-left block $B^{T} B-r I_{n}$ of $A_{G}^{2}-r I_{2 n}$ represents the adjacency matrix of an $r(r-1)$-regular graph, say $H$. Moreover, if $r, \lambda_{2}, \ldots, \lambda_{k}$ are (distinct) non-negative eigenvalues of $G$, then the eigenvalues of $H$ belong to $\left\{r^{2}-r, \lambda_{2}^{2}-r, \ldots, \lambda_{k}^{2}-r\right\}$. 
Therefore, in the search for $G$, we may check whether $H$ exists or not, and if it does not then $G$ does not exist, either. Conversely, if $H$ exists then $G$ can be reconstructed from it. in [9].

Accordingly, we confirm the existence of bipartite 4-regular graphs with 2 spectra listed

Theorem 5.1. There exists a connected bipartite 4-regular graph with data

$$
(15,0,10,4,0,0,210) \text { and }(16,0,12,0,3,0,192)
$$

(both given in the form $\left(n, m_{3}, m_{2}, m_{1}, m_{0}, q, h\right)$, where the parameters are defined in Theorem 4.3).

Proof. Considering the first data for our graph, say $G$, we arrive at a putative 12-regular graph $H$ with 15 vertices and whose eigenvalues belong to $\{12,0,-3\}$. Thus, $H$ is strongly regular (see [6, Theorem 3.4.7]). Moreover, since 0 is one of the eigenvalues, it must be complete multipartite [6, Theorem 3.4.9]. This graph is unique, and we can use it to construct $G$ by obeying the following rules:

- the vertices of $H$ correspond to the vertices of one colour class of $G$;

- the vertices from the same colour class of $H$ do not have common neighbours in $G$;

- every two vertices from different colour classes of $H$ have exactly one common neighbour in $G$.

Finally, $G$ is the incidence graph of a block design with points $1,2, \ldots, 15$ arranged into the following 15 blocks:

$\begin{array}{lllllllllllllllllllll}4 & 7 & 10 & 13 & 13 & 8 & 12 & 13 & 1 & 5 & 10 & 15 & 1 & 6 & 7 & 14 & 1 & 4 & 9 & 11 \\ 5 & 8 & 11 & 14 & 2 & 9 & 10 & 14 & 2 & 6 & 11 & 13 & 2 & 4 & 8 & 15 & & 2 & 5 & 7 & 12 \\ 6 & 9 & 12 & 15 & 3 & 7 & 11 & 15 & 3 & 4 & 12 & 14 & 3 & 5 & 9 & 13 & & 3 & 6 & 8 & 10\end{array}$

The second data is considered in the same way. Now, $H$ has 16 vertices, its eigenvalues belong to $\{12,0,-4\}$, and thus $H$ is again a unique complete multipartite graph. Using the same method, we arrive at $G$ determined by the following blocking:

$\begin{array}{rrrrrrrrrrrrrrrr}1 & 5 & 9 & 13 & 1 & 2 & 7 & 8 & 1 & 3 & 10 & 12 & 1 & 4 & 14 & 15 \\ 2 & 6 & 10 & 14 & 3 & 4 & 5 & 6 & 2 & 4 & 9 & 11 & 2 & 3 & 13 & 16 \\ 3 & 7 & 11 & 15 & 9 & 10 & 15 & 16 & 5 & 7 & 14 & 16 & 5 & 8 & 10 & 11 \\ 4 & 8 & 12 & 16 & 11 & 12 & 13 & 14 & 6 & 8 & 13 & 15 & 6 & 7 & 9 & 12\end{array}$

The proof is complete.

\section{References}

[1] N. Alon, R. Yuster and U. Zwick, Finding and counting given length cycles, Algorithmica 17 (1997), 209-223, doi:10.1007/bf02523189.

[2] F. C. Bussemaker and D. M. Cvetković, There are exactly 13 connected, cubic, integral graphs, Univ. Beograd. Publ. Elektrotehn. Fak. Ser. Mat. Fiz. № 544 - № 576 (1976), 43-48, http : //purl.tue.nl/52815906113922. 
[3] F. Harary and A. J. Schwenk, Which graphs have integral spectra?, in: R. A. Bari and F. Harary (eds.), Graphs and Combinatorics, Springer, Berlin, volume 406 of Lecture Notes in Mathematics, 1974 pp. 45-51, proceedings of the Capital Conference on Graph Theory and Combinatorics at the George Washington University, Washington, D.C., June 18 - 22, 1973.

[4] A. J. Schwenk, Exactly thirteen connected cubic graphs have integral spectra, in: Y. Alavi and D. R. Lick (eds.), Theory and Applications of Graphs, Springer, Berlin, volume 642 of Lecture Notes in Mathematics, 1978 pp. 516-533, proceedings of the International Conference held at Western Michigan University, Kalamazoo, Mich., May 11 - 15, 1976.

[5] S. K. Simić and Z. Stanić, $Q$-integral graphs with edge-degrees at most five, Discrete Math. 308 (2008), 4625-4634, doi:10.1016/j.disc.2007.08.055.

[6] Z. Stanić, Regular Graphs: A Spectral Approach, volume 4 of De Gruyter Series in Discrete Mathematics and Applications, De Gruyter, Berlin, 2017.

[7] Z. Stanić, Perturbations in a signed graph and its index, Discuss. Math. Graph Theory 38 (2018), 841-852, doi:10.7151/dmgt.2035.

[8] Z. Stanić, Bounding the largest eigenvalue of signed graphs, Linear Algebra Appl. 573 (2019), 80-89, doi:10.1016/j.laa.2019.03.011.

[9] D. Stevanović, N. M. M. de Abreu, M. A. A. de Freitas and R. Del-Vecchio, Walks and regular integral graphs, Linear Algebra Appl. 423 (2007), 119-135, doi:10.1016/j.laa.2006.11.026.

[10] T. Zaslavsky, Matrices in the theory of signed simple graphs, in: B. D. Acharya, G. O. H. Katona and J. Nešetřil (eds.), Advances in Discrete Mathematics and Applications, Ramanujan Mathematical Society, Mysore, volume 13 of Ramanujan Mathematical Society Lecture Notes Series, 2010 pp. 207-229, proceedings of the International Conference (ICDM 2008) held at the University of Mysore, Mysore, June 6-10, 2008. 